\title{
Improving the electrical conductivity of carbon fiber reinforced epoxy composite using reduced graphene oxide
}

\begin{abstract}
In this research a reduced graphene oxide ( $\mathrm{rGO}$ ) is used to coat the carbon fiber reinforced epoxy composite (CFRE) to improve and enhance its electrical conductivity that can be used in the aviation applications, because CFRE has poor electrical conductivity, and cannot withstand high electrical current coming from lightning strike. The results show that the electrical conductivity of CFRE is enhanced and increased significantly when it is coated with rGO by about $8015 \%$, where the electrical conductivity of CFRE is increased from $1.38 \times 10^{3}(\mathrm{~S} / \mathrm{m})$ to $1.12 \times 10^{5}(\mathrm{~S} / \mathrm{m})$. Also when the content of rGO increased, the electrical conductivity of CFRE neat will be increased to higher values. The self-heating of all tested specimens was analyzed by the Joule effect. It is found that the self-heating of CFRE is enhanced and improved after coating it with $\mathrm{rGO}$, therefore the self- heating of CFRE become more homogeneous, effective, reaching higher temperatures, than CFRE neat.
\end{abstract}

